\title{
AN ALGORITHM FOR COMPUTING THE VALUES OF THE RAMIFICATION INDEX IN THE PUISEUX SERIES EXPANSIONS OF AN ALGEBRAIC FUNCTION
}

\author{
DAVId LeE HillikeR ${ }^{1}$
}

\begin{abstract}
A quantitative version of a classical algorithm for computing the values of the ramification index of an algebraic function is developed. In our approach the number of steps required to carry out the calculation can be a priori determined.
\end{abstract}

1. Statement of results. We develop in $\S 1$ enough of the foundation and required notation for stating our algorithm for computing the values of the ramification index of an algebraic function. In $\$ 2$ we develop the foundation for calculating each Puiseux series exponent, in reduced form, and the value of the associated coefficient, of such a function. In $\S 3$ we couple these methods with a result of Hilliker and Straus [4] to yield a proof of our algorithm.

Let $F(w, z)$ be any polynomial in two complex variables $w, z$ with coefficients in the field of complex numbers. We consider the algebraic function $w=w(z)$ defined by $F(w, z)=0$. We can view $F(w, z)$ as a polynomial in $w, F(w, z)=f_{n}(z) w^{n}+f_{n-1}(z) w^{n-1}+\cdots+f_{0}(z)$, where $f_{n}(z), f_{n-1}(z), \ldots, f_{0}(z)$ are polynomials in $z$ of maximal degree $m$, with coefficients in the field of complex numbers, and where $f_{n}(z)$ is not identically zero. We assume that $F(w, z)$ is irreducible, over the field of complex numbers, so that in particular, $f_{0}(z)$ is not identically zero. We let $d=\max (m, n)$.

Let $R_{0}$ be a real number large enough so that all finite critical points (singularities, or points with multiple functional values) of the algebraic function $w$ lie in the interior of the circle $|z|=R_{0}$. Let $N\left(R_{0}\right)$ denote the neighborhood of infinity, $|z|>R_{0}$. Thus for each $z$ in $N\left(R_{0}\right)$, the values of the algebraic function are distinct and exactly $n$ in number. Let $R\left(F, F_{w}\right)$ denote the resultant of $F$ and $F_{w}=\partial F / \partial w$, viewed as polynomials in $w$. Thus the finite critical points of $w$ lie among the solutions to the

\footnotetext{
${ }^{1}$ We observe with great regret that Ernst Gabor Straus died on July 12,1983, as a result of a heart attack. Professor Straus was my friend, my Ph.D. thesis advisor, and the coauthor of several of our papers. He did read, and express interest in, a preliminary version of this paper.
} 
polynomial equation $R\left(F, F_{w}\right)=0$, and consequently the quantity $R_{0}$ is readily computable.

According to Puiseux's Theorem, for $z$ in $N\left(R_{0}\right)$, the $n$ distinct values $w(z)$ of the algebraic function $w$ are given by a finite number of Puiseux series

$$
w(z)=\sum_{j=0}^{\infty} c_{j} z^{(p-j) / r} .
$$

Here $p, r$ are integers, $r \geq 1$, and the $c_{j}$ 's are complex, with $c_{0} \neq 0$. There are up to $n$ distinct choices for the ordered set consisting of $p, r, c_{0}, c_{1}, c_{2}, \ldots$, with the various values of $r$, which are not necessarily distinct, adding up to $n$. Each such choice determines a branch of $w$.

The quantity $r$ is called the ramification index of the algebraic function $w$. Systematic methods for computing $r$, which are guaranteed to terminate in a finite number of steps, are known. See Walker [5]. Here we develop an algorithm for computing the ramification index $r$, wherein the number of steps required to carry out the calculations can be a priori determined.

It is convenient to denote the various Puiseux series expansions of $w$ by

$$
w(z)=d_{0} z^{e_{0}}+d_{1} z^{e_{0}+e_{1}}+d_{2} z^{e_{0}+e_{1}+e_{2}}+\cdots,
$$

where $d_{j} \neq 0$ for all $j \geq 0, d_{0}=c_{0}, e_{0}=p / r$, and where the $e_{j}$ 's are rational, with bounded denominators, and with $e_{j}<0$ for $j \geq 1$.

By employing traditional techniques involving Newton's polygon (see $\S 2$ for details) we can calculate the degree $e_{0}=m_{0} / n_{0}, n_{0}>0$, of $w$, as a fraction in reduced form. For each such determination of $e_{0}$, we can substitute the series (2) for $w$ into $F(w, z)=0$. The leading term that so results will have a coefficient $d_{0}^{i_{0}} Q_{0}\left(d_{0}\right)$, where $i_{0}$ is a nonnegative integer and where $Q_{0}$ is a polynomial with $Q_{0}(0) \neq 0$. Thus the values of $d_{0}$ that correspond to this choice of $e_{0}$ are the various nonzero solutions to the equation

$$
z^{i_{0}} Q_{0}(z)=0 .
$$

If $w$ is not identically equal to $d_{0} z^{e_{0}}$, we repeat this process by applying it to

$$
v_{1}=d_{1} z^{e_{1}}+d_{2} z^{e_{1}+e_{2}}+d_{3} z^{e_{1}+e_{2}+e_{3}}+\cdots .
$$

Thus $v_{1}$ is an algebraic function of a formal variable

$$
z_{1}=z^{1 / n_{0}},
$$


related to $w$ by $w=d_{0} z_{1}^{m_{0}}+z_{1}^{m_{0}} v_{1}$. The algebraic function $v_{1}$ satisfies the polynomial equation $F\left(v_{1}, z_{1}\right)=0$, where

$$
F\left(v_{1}, z_{1}\right)=F\left(d_{0} z_{1}^{m_{0}}+z_{1}^{m_{0}} v_{1}, z_{1}^{n_{0}}\right) .
$$

This process can be continued indefinitely. At the $j$ th stage, $j \geq 0$, the values of $e_{j}$ are determined, and for each such determination, the corresponding values of $d_{\text {, }}$ are determined as the nonzero solutions to an equation

$$
z^{i} Q_{J}(z)=0, \quad j \geq 0,
$$

where $i_{j}$ is a nonnegative integer and where $Q_{J}(z)$ is a polynomial with $Q_{j}(0) \neq 0$.

Our algorithm, the main result of this paper, can now be formulated:

THEOREM. If there is a value of $j<2 d^{3}-d^{2}+d$ for which the nonzero solutions to (3) are all simple, let $j=j_{1} \geq 0$ be the smallest such value; otherwise, let $j_{1}=2 d^{3}-d^{2}+d$. Then the various values of the ramification index $r$ are the various values of the least common denominator of $e_{0}, e_{1}$, $e_{2}, \ldots, e_{j_{1}}$.

2. Fundamental calculations. We shall employ a version of Newton's polygon. Let $w$ be any function, not necessarily algebraic, of finite degree $\alpha$, so that $|w| /|z|^{\alpha}$ has a finite nonzero limit as $z$ approaches infinity. We consider our fixed polynomial

$$
F(w, z)=f_{n}(z) w^{n}+f_{n-1}(z) w^{n-1}+\cdots+f_{0}(z),
$$

with $f_{n}(z)$ and $f_{0}(z)$ not identically zero. In this discussion, $w$ varies over the set of all functions of finite degree of a complex variable $z$. Let $x, y$ be real numbers defined by $x=\operatorname{deg} w, y=\operatorname{deg} F(w, z)$. Then $y$ is a function of $x$ defined over the entire real line. One has

$$
y \leq \max \left(\operatorname{deg} f_{n}(z)+n x, \operatorname{deg} f_{n-1}(z)+(n-1) x, \ldots, \operatorname{deg} f_{0}(z)\right) .
$$

The points $(x, y)$ in the $x y$-plane lie in a certain region, consisting of all points on or below a certain polygon. This polygon, which we shall call Newton's polygon, consists of at least two, and at most $n+1$ line segments selected from lines of the form $y=\operatorname{deg} f_{j}(z)+j x$, for certain values of $j$ in the range $0 \leq j \leq n$. The first line segment, going from left to right, is given by $j=0$. The last line segment is given by $j=n$. The polygon is concave upword. The vertices of this polygon have rational coordinates. If $x$ is not the coordinate of a vertex, then equality in (4) holds. 
We now focus attention on the case where $w$ is our previous algebraic function. If we substitute the Puiseux series (1) for $w$ into $F(w, x)=0$, we see that there will be at least two terms of leading degree, and these must cancel. This implies that the degree of the branch $w(z)$, given by (1), is the $x$-coordinate of some vertex. The coefficients of these terms of leading degree must add up to zero. Thus the values of $c_{0}$ are the nonzero solutions to the equation

$$
\sum g_{h} z^{h}=0
$$

where $g_{h}$ is the leading coeffcient in $f_{h}(z)$, for $0 \leq h \leq n$, and where the summation is taken under those values of $h$ in the range $0 \leq h \leq n$ for which $\operatorname{deg} f_{h}(z)+h x=y$, where $(x, y)$ is the vertex under consideration. Note that (5) always has a nonzero solution, since $h$ always takes on at least two values.

We have realized the following: The degree of each branch of the algebraic function $w$ is the $x$-coordinate of some vertex of Newton's polygon and the values of the leading coefficient that correspond to this degree are given by (5). Conversely, it can be shown that: The $x$-coordinate of each vertex is the degree of one or more branches of the algebraic function $w$.

Newton's polygon affords us a practical method for computing the values of the ratio $p / r$, and for each such value, the corresponding values of $c_{0}$. It does, as we have already suggested, provide us with a method of computing all branches of the algebraic function $w$, out to as many terms as is required. We use the notation (2).

First, we compute $\operatorname{deg} w=e_{0}=m_{0} / n_{0}$, as a rational number in reduced form, with $n_{0}>0$, from Newton's polygon for $F(w, z)$, and for each such value of $e_{0}$, we compute the corresponding values of the leading coefficient $d_{0}$, from the equation (5). We then make a formal substitution $z_{1}=z^{1 / n_{0}}$ and introduce a Puiseux series $v_{1}$ by $w=d_{0} z_{1}^{m_{0}}+z_{1}^{m_{0}} v_{1}$. Then, $v_{1}$ represents an algebraic function of $z_{1}$ defined by $F_{1}\left(v_{1}, z_{1}\right)=0$ where $F_{1}\left(v_{1}, z_{1}\right)$ is a polynomial given by

$$
F_{1}\left(v_{1}, z_{1}\right)=F\left(d_{0} z_{1}^{m_{0}}+z_{1}^{m_{0}} v_{1}, z_{1}^{n_{0}}\right) .
$$

If $v_{1}$ is identically zero, the process stops here and $w=d_{0} z^{e_{0}}$. Otherwise, we compute, from Newton's polygon for $F_{1}\left(v_{1}, z_{1}\right), \operatorname{deg}_{z_{1}} v_{1}$, and for each such value we compute the associated values of the leading coefficient $d_{1}$ of $v_{1}$, from an equation analogous to (5). The only difference in this second application of Newton's polygon is that we restrict attention to 
those vertices with negative $x$-coordinates. Then, one has a determination of

$$
\operatorname{deg}_{z} v_{1}=\frac{1}{n_{0}} \operatorname{deg}_{z_{1}} v_{1}=e_{1}=\frac{m_{1}}{n_{1}},
$$

where $m_{1} / n_{1}$ is in reduced form with $n_{1}>0$. We then set

$$
z_{2}=z_{1}^{1 / n_{1}} \text { and } v_{1}=d_{1} z_{2}^{m_{1}}+z_{2}^{m_{1}} v_{2},
$$

so that $v_{2}$ is an algebraic function of $z_{2}$ defined by

$$
F_{2}\left(v_{2}, z_{2}\right)=F_{1}\left(d_{1} z_{2}^{m_{1}}+z_{2}^{m_{1}} v_{2}, z_{2}^{n_{1}}\right)=0 .
$$

If $v_{2}$ is not identically zero, we compute $\operatorname{deg}_{z_{2}} v_{2}$ and for each such determination we compute the corresponding values of the leading coefficient $d_{2}$ of $v_{2}$. We then have a determination of

$$
\operatorname{deg}_{z} v_{2}=\frac{1}{n_{0} n_{1}} \operatorname{deg}_{z_{2}} v_{2}=e_{2}=\frac{m_{2}}{n_{2}},
$$

in reduced form with $n_{2}>0$.

This process can be continued indefinitely. In general terms, we have for each $i \geq 0$, an algebraic function $v_{i}=v_{i}\left(z_{i}\right)$ with $v_{0}=w, z_{0}=z$, given recursively by

$$
v_{i-1}=d_{i-1} z_{i}^{m_{t-1}}+z_{i}^{m_{i-1}} v_{i}
$$

with, formally,

$$
z_{i}=z_{i-1}^{1 / n-1}
$$

The quantity

$$
\operatorname{deg}_{z_{i}} v_{i}=n_{0} n_{1} n_{2} \cdots n_{i-1} \operatorname{deg}_{z} v_{i}=n_{0} n_{1} n_{2} \cdots n_{i-1} e_{i}
$$

can be determined from Newton's polygon. Consequently, $e_{i}=m_{i} / n_{i}$ can be determined as a reduced fraction with $n_{i}>0$, and for each such determination, the corresponding values of the leading coefficient $d_{i}$ of $v_{i}$ can be determined from an equation analogous to (5). The defining polynomial equation for $v_{i}$ is $F_{i}\left(v_{i}, z_{l}\right)=0$ where

$$
F_{i}\left(v_{i}, z_{i}\right)=F_{i-1}\left(d_{i-1} z_{i}^{m_{t-1}}+z_{i}^{m_{t-1}} v_{i}, z_{i}^{n_{t-1}}\right),
$$

is of degree $n$ in $v_{i}$, and where $F_{0}=F$. Later we shall use the notation

$$
F_{i}\left(v_{i}, z_{i}\right)=f_{n}\left(z_{i} ; i\right) v_{\imath}^{n}+f_{n-1}\left(z_{i} ; i\right) v_{i}^{n-1}+\cdots+f_{0}\left(z_{i} ; i\right)
$$

where for each $k \geq 0$, the polynomial $f_{n-k}\left(z_{i} ; i\right)$, which reduces to $f_{n-k}(z)$ when $i=0$, has degree $m_{l, k}$ in $z_{i}$ and leading coefficient $a_{m_{l, k}}$. 
3. Determination of the ramification index. The techniques of $\S 2$ furnish us with an algorithm for computing all Puiseux expansions of the algebraic function $w$ out to as many terms as is required, but so far we have not indicated a method for computing the various values of the quantity $r$. We develop such an algorithm here, in three stages.

First, we draw upon a result (Theorem 4.5) established in the paper of Hilliker and Straus [4]: There is a value of the index $j$, and so a smallest value $j=j_{0} \geq 0$, so that if $j>j_{0}$, the equation for $c_{j}$, obtained by substituting the Puiseux series expansion for $w$ into $F(w, z)=0$, is linear, of the form $A c_{j}+B_{J}=0$, where $A \neq 0$ is a polynomial in $c_{0}, c_{1}, \ldots, c_{j_{0}}, B_{j}$ is a polynomial in $c_{0}, c_{1}, \ldots, c_{j-1}$, and where the coefficients in $A$ and $B$ are field elements in the field generated by the coefficients of $F(w, z)$. A sufficient condition for $j_{0}=0$ is that

$$
\sum(n-k) a_{m_{k}} c_{0}^{n-k} \neq 0,
$$

where $m_{k}$, for $0 \leq k \leq n$, is the degree of $f_{n-k}(z), a_{m_{k}}$ is the leading coefficient in $f_{n-k}(z)$, and where the summation in (8) is taken under those values of $k$ for which

$$
m_{k}+\frac{p}{r}(n-k)
$$

is maximal. In any case,

$$
j_{0} \leq 2 d^{3}-d^{2}+d,
$$

where $d=\max (m, n)$. In particular, all the Puiseux coefficients lie in the field obtained by adjoining $c_{0}, c_{1}, \ldots, c_{j_{0}}$ to the field of the coefficients of $F(w, z)$.

Note that equation (5) for $c_{0}$ can be written as:

$$
\sum a_{m_{k}} z^{n-k}=0
$$

where the summation is extended over the same values of $k$ as those of (8). Consequently, (8) is precisely the condition that the equation for $c_{0}$, obtained by substituting the Puiseux series for $w$ into $F(w, z)=0$, has only simple solutions.

Secondly, we establish, here, the following: If the equation for the leading Puiseux coefficient, obtained by substituting the Puiseux series expansion for $w$ into $F(w, z)=0$, is linear, then the branch under consideration has an integral degree.

To prove this we use the notation of (1). We let $(x, y)$ be the vertex of Newton's polygon that corresponds to the branch of $w$ under consideration. Suppose that $(x, y)$ is determined as the intersection of the two lines

$$
y=\operatorname{deg} f_{j}(z)+j x \text { and } y=\operatorname{deg} f_{k}(z)+k x,
$$


with $k>j$, each of which lie on a segment of Newton's polygon. Then, the degree of $w$ is given by

$$
x=\frac{\operatorname{deg} f_{j}(z)-\operatorname{deg} f_{k}(z)}{k-j}=\frac{u}{v},
$$

where $u / v$ is the reduced form of this ratio, and where $v>0$. Thus, $v$ divides $k-j$. If

$$
y=\operatorname{deg} f_{h}(z)+h x
$$

is any such line that passes through the vertex under consideration, then, since the lines $y=\operatorname{deg} f_{j}(z)+j x$ and $y=\operatorname{deg} f_{k}(z)+k x$ both lie on Newton's polygon, $h$ lies in the range $j \leq h \leq k$ and the values $h=j$ and $h=k$ are actually realized. Moreover, we can determine the vertex $(x, y)$ as the intersection of the lines $y=\operatorname{deg} f_{j}(z)+j x$ and $y=\operatorname{deg} f_{h}(z)+h x$, provided $h>j$. That is to say, we get, from a formula analogous to (10) with $k$ on the left side replaced by $h$, that $v$ divides $h-j$, so that $h=j+s v$, for some positive integer $s$. If $h=j$, this formula still holds with $s=0$. We realize that $h$ in (11) is of the form

$$
h=j+s v \text {. }
$$

In (12) $j$ and $v$ are fixed integers and $s$ takes on certain nonnegative integral values. It follows from (12) that the polynomial $\sum g_{h} x^{h}$, on the left side of (5), has the form

$$
\sum g_{h} z^{h}=z^{j} P\left(z^{v}\right)
$$

where $P(t)$ is a polynomial with $P(0) \neq 0$, since the minimal degree of the various terms on the left of (13) is $j$, and with $\operatorname{deg}_{t} P(t)=(k-j) / v$, since the polynomial on the left of (13) is of degree $k$ in $z$.

We have shown the following: The equation for the leading coefficient $c_{0}$ obtained by substituting the Puiseux series (1) for $w$ into $F(w, z)=0$, is of the form

$$
z^{j} P\left(z^{v}\right)=0,
$$

where $j$ is a nonnegative integer, where the reduced form of the degree of the branch under consideration is $u / v$ with $v>0$, and where $P$ is a polynomial with $P(0) \neq 0$.

Our proof is now complete, since the polynomial equation (14) could be linear only if $v=1$ so that $x=\operatorname{deg} w=u$ is an integer.

Note that the degree in $c_{0}$ of the polynomial $P\left(c_{0}^{v}\right)$ is the positive change in the slope at the vertex. 
Thirdly, we employ the notation of (2) and construct the algebraic functions $v_{j}$ in accordance with (6). Condition (8) can be applied to the algebraic functions $v_{j}$, for $j \geq 0$, using the notation of (7):

$$
\sum(n-k) a_{m_{j, k}} d_{j}^{n-k} \neq 0,
$$

where the summation is taken under those values of $k, 0 \leq k \leq n$, for which

$$
m_{j, k}+\frac{p}{r}(n-k)
$$

is maximal.

It is observed that the equation for $d_{j}$, obtained by substituting the Puiseux series (2) for $w$ into $F(w, z)=0$, is the same as the equation for $d_{j}$ obtained by substituting the Puiseux series for $v_{j}=v_{j}\left(z_{j}\right)$, with leading coefficient $d_{j}$, into $F_{j}\left(v_{j}, z_{j}\right)=0$. This equation for $d_{j}$ is:

$$
\sum a_{m_{j, k}} d_{j}^{n-k}=0,
$$

where the summation is taken under those same values of $k$ as in (15). Accordingly, we conclude that (15) is a necessary and sufficient condition for the equation for $d_{j}$ to have only simple roots, for a fixed choice of $d_{0}, d_{1}$, $d_{2}, \ldots, d_{j-1}$. Moreover, it is the case that (15) holds for all sufficiently large values of $j$, for then, the equation for $d_{j}$ would be linear, since $d_{j}=c_{i}$, for some $i \geq j$.

We can now state our algorithm, the main result of this paper, in terms more precise than those of $\$ 1$, in view of (9):

THEOREM. If there is a value of $j<2 d^{3}-d^{2}+d$ for which (15) holds, let $j=j_{1} \geq 0$ be the smallest such value; otherwise, let $j_{1}=2 d^{3}-d^{2}+d$. Then, the various values of the ramification index $r$ are the various values of the least common denominator of $e_{0}, e_{1}, e_{2}, \ldots, e_{j_{1}}$.

To illustrate the ideas, let us compute the ramification index of the algebraic function $w$ given by $w^{3}-(1+3 z) w^{2}+3 z^{2} w-z^{3}+z=0$. Newton's polygon has only one vertex, namely, $(1,3)$. Thus all branches are of degree 1 . The equation (14) for the leading coefficient is $\left(d_{0}-1\right)^{3}$ $=0$, so that $d_{0}=1$ with multiplicity 3 . Condition (8) fails for $w$. The algebraic function $v_{1}$ of $z_{1}=z$ is given by $w=z+z v_{1}$, and has the defining equation $z^{3} v_{1}^{3}-z^{2} v_{1}^{2}-2 z^{2} v_{1}-z^{2}+z=0$. All branches of $v_{1}$ are of degree $-1 / 3$. The equation (14) for the leading coefficient of $v_{1}$ is $d_{1}^{3}-1=0$. Condition (15) holds for $v_{1}$ so that $j_{1}=1$. We now conclude that $r$ takes on only the value $r=3$. 
For more on Puiseux series, and their applications to Diophantine equations, see the papers of Hilliker [1], [2]; and those of Hilliker and Straus [3], [4].

\section{REFERENCES}

[1] David Lee Hilliker, An algorithm for solving a certain class of Diophantine equations, $I$, Math. Comp., 38 (1982), 611-626.

[2] __ An algorithm for solving a certain class of Diophantine equations, II, to be submitted.

[3] David Lee Hilliker and E. G. Straus, On Puiseux series whose curves pass through an infinity of algebraic lattice points, Bull. Amer. Math. Soc., (N.S.) 8 (1983), 59-62.

[4] Determination of bounds for the solutions to those binary Diophantine equations that satisfy the hypotheses of Runge's theorem, Trans. Amer. Math. Soc., 280 (1983), 637-657.

[5] Robert J. Walker, Algebraic Curves, Princeton University Press, Princeton, New Jersey, 1950. Reprinted by Dover, New York, 1962.

Received May 22, 1984 and in revised form October 15, 1984.

California State University

LONG BEACH, CA 90840 
